\title{
On Best Corrected Mixture Problems in Metallurgy: A Case Study
}

\author{
F. Dubeau and M. E. Ntigura Habingabwa \\ Département de Mathématiques, Université de Sherbrooke, 2500 Boulevard de l'Université, Sherbrooke, QC, Canada J1K 2R1 \\ Correspondence should be addressed to F. Dubeau; francois.dubeau@usherbrooke.ca
}

Received 15 October 2016; Revised 28 January 2017; Accepted 30 January 2017; Published 27 February 2017

Academic Editor: Risto Lahdelma

Copyright (C) 2017 F. Dubeau and M. E. Ntigura Habingabwa. This is an open access article distributed under the Creative Commons Attribution License, which permits unrestricted use, distribution, and reproduction in any medium, provided the original work is properly cited.

\begin{abstract}
We consider three simple mixture problems occurring in metallurgy. The first problem considered is the classic minimum cost mixture problem. For the second problem, we consider finding a correction to a given mixture, a premix, without considering the cost of this premix. We only consider the cost and the weight of the quantity used as a correction. We show that the minimum cost correction does not correspond to the minimum weight correction, and we built the Pareto curve that gives all intermediate solutions between these two extreme solutions. Finally, the third problem is the correction problem for a nonfree premix. The correction is done to obtain a minimum cost corrected mixture.
\end{abstract}

\section{Introduction}

Using materials, such as scrap metal for recycling, in order to form blends with desired specifications on some basic chemical elements is a well-known mixture problem met in foundries. People working in these fields bring to our attention the three different but related problems that we are going to consider in this paper. The first problem, called the basic problem, is to make a minimum cost mixture while checking the constraints on the specifications. It is a classic mixture problem solved by linear programming. For the second problem, called the correction problem, we start with a given mixture, called here a premix, which does not satisfy all the constraints on the specifications. Without considering the cost of this premix, we look for a correction, which is itself a mixture of available materials, that we will add to the premix in such a way that the resulting corrected mixture, which is the premix and the correction added together, satisfies all the constraints on the specifications. The correction can be done at minimum cost or at minimum weight. This problem is an example of a bicriteria linear programming problem, and we are going to identify its Pareto set which gives the link between minimum cost solution and minimum weight solution. Finally, the third problem is similar to the preceding correction problem, but now we take into account the cost of the given premix. We look for a minimum unit cost corrected mixture. This is an example of a linear-fractional programming problem. It is important to point out that the questions raised in this paper and the data used in the examples are inspired from a real situation.

Mixture problems are important applications in Operations Research [1]. One such application is the diet problem which received a lot of attention [2-7]. A variant of this problem is the pooling problem [8]. Also other approaches could be used to analyze similar mixture problems; for example, the fuzzy approach could be used to introduce uncertainties on the data [9].

\section{Basic Problem}

2.1. Problem Description. For the basic problem, there are $N$ materials that can be used to produce a mixture. Let $\pi_{n} \geq 0$ be the weight of the $n$th material in the final mixture $(n=$ $1, \ldots, N)$. The total weight of the mixture is

$$
z_{\text {weight }}(\pi)=\sum_{n=1}^{N} \pi_{n}
$$


The total cost of the mixture of weight $z_{\text {weight }}$ is then

$$
z_{\text {cost }}(\pi)=\sum_{n=1}^{N} c_{n} \pi_{n},
$$

where $c_{n} \geq 0$ is the unit costs of the $n$th material (for $n=$ $1, \ldots, N)$.

The specifications of the mixture and of the materials are the amount (proportion \% of the content) of known basic chemical elements (aluminium, carbon, chromium, copper, iron, etc.) contained in a unit weight of the material. Typically, there may be between 10 and 20 basic chemical elements that characterize a chemical mixture. Each $n$th material is characterized by $M$ specifications noted by $s_{m, n}(m=$ $1, \ldots, M)$.

The specifications of the mixture are determined by a linear combination of the specifications of the $N$ elements of the mixture. If we denote by spec $_{m}$, the $m$ th specification of the mixture, we have

$$
\operatorname{spec}_{m} z_{\text {weight }}(\pi)=\sum_{n=1}^{N} s_{m, n} \pi_{n} .
$$

The specifications of the mixture are subjected to two types of constraints.

Type 1. Each specification must be in a well-defined range; that is to say,

$$
\operatorname{spec}_{m}^{\min } \leq \operatorname{spec}_{m} \leq \operatorname{spec}_{m}^{\max }
$$

for $m=1, \ldots, M$, where $\operatorname{spec}_{m}^{\min } \geq 0$ and $\operatorname{spec}_{m}^{\max } \leq 1$ are, respectively, the minimum and maximum value allowed for the $m$ th specification of the mixture.

Type 2. Specifications can be connected or related by $J$ linear relations of the form

$$
\sum_{m=1}^{M} \alpha_{j, m} \operatorname{spec}_{m}\left\{\begin{array}{l}
\leq \\
= \\
\geq
\end{array}\right\} \beta_{j}
$$

for $j=1, \ldots, J$. For example, in Figure 1, we illustrate a situation where there are 4 links between the specifications of two chemical elements denoted as $A$ and $B$. The pair of specifications $\left(\operatorname{spec}_{A}, \operatorname{spec}_{B}\right)$ must be within the region bounded by the 4 given straight lines.

2.2. Problem Formulation. The basic problem is to look for a minimum cost mixture of unit weight; that is to say, $z_{\text {weight }}(\pi)=1$, satisfying constraints of Types 1 and 2 . The decision variables are $\pi_{n}$ for $n=1, \ldots, N$; they will then be the proportions of each ingredient in the unit weight mixture. The mathematical formulation is therefore

$$
\begin{array}{cc}
\min & z_{\text {cost }}(\pi)=\sum_{n=1}^{N} c_{n} \pi_{n}, \\
\text { subjet to } & \text { (i) } \sum_{n=1}^{N} \pi_{n}=1 ; \\
& \text { (ii) for } m=1, \ldots, M \text { : }
\end{array}
$$

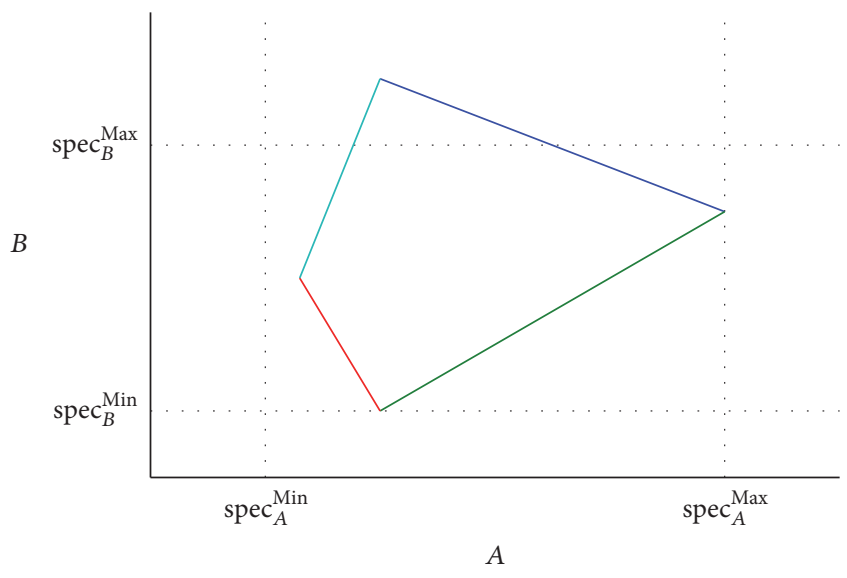

FIGURE 1: Related specifications.

$$
\begin{aligned}
& \sum_{n=1}^{N} s_{m, n} \pi_{n}\left\{\begin{array}{l}
\leq \operatorname{spec}_{m}^{\max }, \\
\geq \operatorname{spec}_{m}^{\min } ;
\end{array}\right. \\
& \text { (iii) for } j=1, \ldots, J: \\
& \sum_{n=1}^{N}\left[\sum_{m=1}^{M} \alpha_{j, m} s_{m, n}\right] \pi_{n}\left\{\begin{array}{l}
\leq \\
=
\end{array}\right\} \beta_{j} ; \\
& \text { (iv) } \pi_{n} \geq 0 \text { for } n=1, \ldots, N .
\end{aligned}
$$

Let $\mathcal{S}$ be the notation for the set of feasible solutions to this problem.

2.3. Numerical Example. In our numerical example coming from a real context, we have $N=8$ materials and the mixture has specifications on $M=11$ chemical elements. The minimum and maximum values for the specifications of the mixture are listed in Table 1 . We have 2 specifications on 11 basic chemical elements; then there are 22 constraints concerning the concentration of the basic chemical elements in the mixture. Specifications and costs of the 8 materials are given in Table 2 .

Constraints of the first type are completely determined using the data in Tables 1 and 2. Constraints of the second type require some clarification. We consider 4 constraints of Type 2 connecting carbon $(m=2)$ and chromium $(m=$ 3) specifications. Constraints are described geometrically in Figure 2. They indicate that the pair $\left(\mathrm{spec}_{2}, \mathrm{spec}_{3}\right)$ should be inside the parallelogram enclosed by 4 straight lines. With the data provided, the constraints are

$$
\begin{aligned}
& \text { line no. } 1: \mathrm{spec}_{3}-\frac{50}{9} \text { spec }_{2} \leq 9.2500 \\
& \text { line no. } 2: \mathrm{spec}_{3}-\frac{50}{9} \mathrm{spec}_{2} \geq 7.6944 \\
& \text { line no. } 3: \mathrm{spec}_{3}+10 \mathrm{spec}_{2} \leq 54.05 \\
& \text { line no. } 4: \mathrm{spec}_{3}+10 \mathrm{spec}_{2} \geq 51.25 .
\end{aligned}
$$

The coefficients of these constraints are given in Table 3. 
TABLE 1: Minimum and maximum specifications in $\%$ of weight in the mixture.

\begin{tabular}{|c|c|c|c|c|}
\hline \multirow[b]{2}{*}{$m$} & \multicolumn{3}{|c|}{ Specifications (\%) for $M=11$ chemical element of the mixture } & \multirow[b]{2}{*}{$\operatorname{spec}_{m}^{\max }$} \\
\hline & & ement & $\operatorname{spec}_{m}^{\min }$ & \\
\hline 1 & $\mathrm{Al}$ & Aluminium & 0 & 0.2 \\
\hline 2 & $\mathrm{C}$ & Carbon & 2.7 & 2.98 \\
\hline 3 & $\mathrm{Cr}$ & Chromium & 23.25 & 25.25 \\
\hline 4 & $\mathrm{Cu}$ & Copper & 0 & 0.2 \\
\hline 5 & $\mathrm{Fe}$ & Iron & 65 & 85 \\
\hline 6 & $\mathrm{Mn}$ & Manganese & 0.3 & 0.6 \\
\hline 7 & Mo & Molybdenum & 0 & 0.3 \\
\hline 8 & $\mathrm{Ni}$ & Nickel & 0 & 0.2 \\
\hline 9 & $\mathrm{P}$ & Phosphorus & 0 & 0.06 \\
\hline 10 & S & Sulphur & 0 & 0.06 \\
\hline 11 & $\mathrm{Si}$ & Silicone & 0.3 & 0.8 \\
\hline
\end{tabular}

TABLE 2: Characteristics of available materials.

\begin{tabular}{|c|c|c|c|c|c|c|c|c|}
\hline \multicolumn{9}{|c|}{$s_{m, n}$ specifications (\%) of $N=8$ available materials } \\
\hline \multirow[b]{2}{*}{$m$} & \multicolumn{8}{|c|}{$n$} \\
\hline & 1 & 2 & 3 & 4 & 5 & 6 & 7 & 8 \\
\hline 1 & 0 & 1 & 0 & 0 & 0 & 0.0096 & 0.0340 & 0 \\
\hline 2 & 0.2744 & 0 & 80 & 7.2096 & 1.7184 & 6.5088 & 0.0768 & 0 \\
\hline 3 & 0.1666 & 0 & 0 & 48.2880 & 51.9552 & 0 & 0 & 0 \\
\hline 4 & 0.1666 & 0 & 0 & 0 & 0 & 0 & 0 & 0 \\
\hline 5 & 96.1772 & 0 & 0 & 40.6650 & 42.1304 & 15.2928 & 21.7728 & 69.1200 \\
\hline 6 & 0.8232 & 0 & 0 & 0 & 0 & 73.2768 & 0.1632 & 0 \\
\hline 7 & 0.0490 & 0 & 0 & 0 & 0 & 0 & 0 & 0 \\
\hline 8 & 0.1862 & 0 & 0 & 0 & 0 & 0 & 0 & 0 \\
\hline 9 & 0.0392 & 0 & 0 & 0.0192 & 0.0192 & 0.2688 & 0.0096 & 0 \\
\hline 10 & 0 & 0 & 0 & 0.0384 & 0.0192 & 0.0096 & 0 & 0 \\
\hline \multirow[t]{3}{*}{11} & 0.1176 & 0 & 0 & 2.6592 & 0.0672 & 0.5952 & 17.1616 & 0.0900 \\
\hline & \multicolumn{8}{|c|}{ Material unit cost $c_{n}$} \\
\hline & 0.1450 & 1.3500 & 0.1100 & 0.5050 & 0.6750 & 0.5760 & 0.3715 & 0.7250 \\
\hline
\end{tabular}

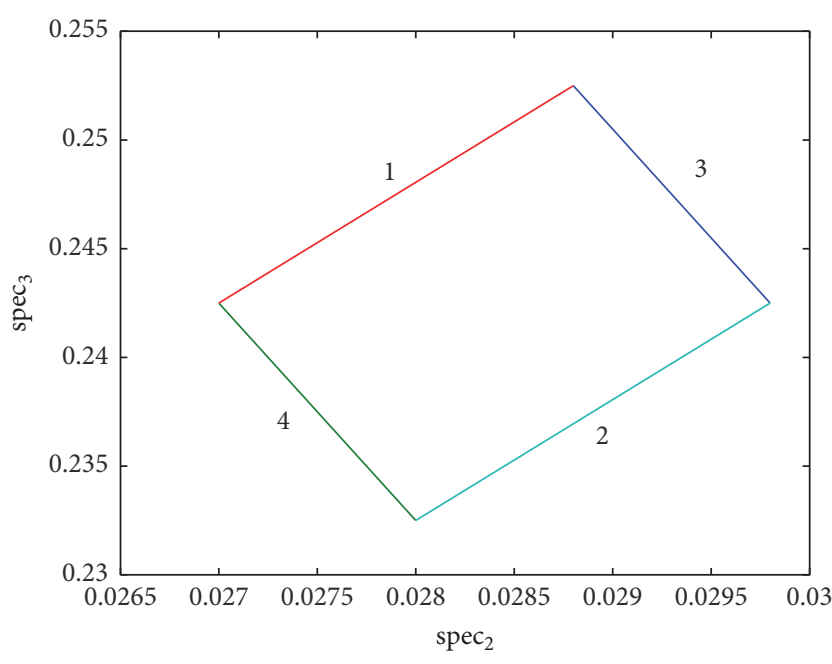

FIGURE 2: Coupled specifications of carbon and chromium.
For the problem $(P)$, the constraints (i) and (iv) ensure that the set of feasible solutions is bounded if it is not empty. This set is actually not empty and we get the optimal solution of Table 4 , with (minimum) unit cost equal to $0.344984 \$$. If we take away the materials $n=5$ and $n=6$, set $\pi_{5}=0$ and $\pi_{6}=0$ in the model, this new problem has no solution, and the feasible set is empty. It means that it is impossible to make a mixture which satisfies all the constraints with the 6 remaining materials.

\section{Correction to a Fixed Quantity of "Free" Premix}

3.1. Problem Description. For the second problem we assume that we have in stock a given mixture already done called a premix, with its own specifications, but which does not satisfy some constraints for a new mixture. The problem we consider is to add to this premix a correction, which is a combination 
TABle 3: Coefficients to Type 2 constraints.

$$
J=4 \text { constraints of Type } 2
$$

\begin{tabular}{|c|c|c|c|c|c|c|c|c|c|}
\hline$j$ & 1 & 2 & 3 & 4 & 5 & 6 & 7 & 8 & $\beta_{j}$ \\
\hline $\begin{array}{l}1 \\
2 \\
\end{array}$ & -1.3578 & 0 & -444.4444 & 8.2347 & 42.4085 & -36.1600 & -0.4267 & 0 & $\begin{array}{l}\leq 9.2500 \\
\geq 7.6944\end{array}$ \\
\hline $\begin{array}{l}3 \\
4\end{array}$ & 2.9106 & 0 & 800 & 120.3840 & 69.1392 & 65.0880 & 0.7680 & 0 & $\begin{array}{l}\leq 54.0500 \\
\geq 51.2500\end{array}$ \\
\hline
\end{tabular}

TABLE 4: Optimal solution of the basic mixture problem.

\begin{tabular}{lccccccc}
\hline$\pi_{1}$ & $\pi_{2}$ & $\pi_{3}$ & $\pi_{4}$ & $\pi_{5}$ & $\pi_{6}$ & $\pi_{7}$ & $\pi_{8}$ \\
\hline 0.530478 & 0 & 0.004486 & 0.272517 & 0.192517 & 0 & 0 \\
\hline
\end{tabular}

of the $N$ available materials, in such a way that the corrected mixture obtained by adding the correction to the premix satisfies all the new constraints on the specifications. We are going to obtain this correction without considering the cost of the premix.

Let us set

$$
\begin{aligned}
& \pi_{0}^{a} \geq 0 \text { for the weight of the premix in the corrected mixture, } \\
& \pi_{n}^{a} \geq 0 \text { for the weight of the } n \text {th material in the corrected mixture }(n=1, \ldots, N) .
\end{aligned}
$$

As we want to correct a given weight of the premix, we set $\pi_{0}^{a}=1$, and the decision vector will be $\pi^{a}=\left(\pi_{1}^{a}, \ldots, \pi_{N}^{a}\right)$.

The weight of the correction to add to the premix to obtain an acceptable mixture is given by

$$
z_{\text {weight }}\left(\pi^{a}\right)=\sum_{n=1}^{N} \pi_{n}^{a}
$$

If we consider a minimum weight correction, we have to minimize $z_{\text {weight }}\left(\pi^{a}\right)$. The total weight of the corrected mixture is given by the following formula:

$$
z_{\text {weight }}^{\text {total }}\left(\pi^{a}\right)=\sum_{n=0}^{N} \pi_{n}^{a}=1+z_{\text {weight }}\left(\pi^{a}\right) \text {. }
$$

The cost of the correction is

$$
z_{\text {cost }}\left(\pi^{a}\right)=\sum_{n=1}^{N} c_{n} \pi_{n}^{a}
$$

If we consider a minimum cost correction, we have to minimize $z_{\text {cost }}\left(\pi^{a}\right)$.

The $M$ specifications of the premix are denoted by $s_{m, 0}(m=1, \ldots, M)$, and each material is also characterized by the $M$ specifications previously described and denoted by $s_{m, n}(m=1, \ldots, M)$. The specifications $\operatorname{spec}_{m}$ of the resulting corrected mixture are then defined by

$$
\operatorname{spec}_{m} z_{\text {weight }}^{\text {total }}\left(\pi^{a}\right)=\sum_{n=0}^{N} s_{m, n} \pi_{n}^{a}=s_{m, n}+\sum_{n=1}^{N} s_{m, n} \pi_{n}^{a},
$$

and the constraints of Types 1 and 2 are directly applicable.
3.2. Problem Formulation. Since the problem we consider is to correct a unit weight of a premix $\left(\pi_{0}^{a}=1\right)$ by adding to that premix a minimum cost or a minimum weight correction, the mathematical formulation is

$$
\begin{aligned}
& \min z_{\text {cost }}\left(\pi^{a}\right)=\sum_{n=1}^{N} c_{n} \pi_{n}^{a}, \\
& \min z_{\text {weight }}\left(\pi^{a}\right)=\sum_{n=1}^{N} \pi_{n}^{a},
\end{aligned}
$$

subjet to (i) $\sum_{n=1}^{N} \pi_{n}^{a}=\pi_{\text {tot }}^{\mathrm{a}}$;

(ii) for $m=1, \ldots, M$ :

$\sum_{n=0}^{N} s_{m, n} \pi_{n}^{a}-\operatorname{spec}_{m}^{\max } \pi_{\text {tot }}^{a} \leq \operatorname{spec}_{m}^{\max }$

$\sum_{n=0}^{N} s_{m, n} \pi_{n}^{a}-\operatorname{spec}_{m}^{\min } \pi_{\text {tot }}^{a} \geq \operatorname{spec}_{m}^{\min }$,

(iii) for $j=1, \ldots, J$ :

$\sum_{n=0}^{N}\left[\sum_{m=1}^{M} \alpha_{j, m} s_{m, n}\right] \pi_{n}^{a}-\beta_{j} \pi_{\text {tot }}^{a}\left\{\begin{array}{l}\leq \\ = \\ \geq\end{array}\right\} \beta_{j}$

(iv) $\pi_{0}^{a}=1$,

$\pi_{n}^{a} \geq 0 \quad$ for $n=1, \ldots, N$. 
Table 5: Premix specifications in \% of weight.

\begin{tabular}{|c|c|c|c|c|c|}
\hline \multirow{3}{*}{$\frac{m}{1}$} & \multicolumn{4}{|c|}{ spec $_{m, 0}$ specifications (\%) of the premix } & \multirow{3}{*}{$\frac{\mathrm{spec}_{m}^{\mathrm{ma} a}}{0.2}$} \\
\hline & \multicolumn{2}{|c|}{ Chemical elements } & \multirow{2}{*}{$\frac{\operatorname{spec}_{m}^{\min }}{0}$} & \multirow{2}{*}{$\begin{array}{l}s_{m, 0} \\
0.15\end{array}$} & \\
\hline & $\mathrm{Al}$ & Aluminium & & & \\
\hline 2 & $\mathrm{C}$ & Carbon & 2.7 & 2.98 & 2.98 \\
\hline 3 & $\mathrm{Cr}$ & Chrome & 23.25 & 25.25 & 25.25 \\
\hline 4 & $\mathrm{Cu}$ & Copper & 0 & 0.19 & 0.2 \\
\hline 5 & $\mathrm{Fe}$ & Iron & 65 & 80 & 85 \\
\hline 6 & $\mathrm{Mn}$ & Manganese & 0.3 & 0.55 & 0.6 \\
\hline 7 & Mo & Molybdenum & 0 & 0.25 & 0.3 \\
\hline 8 & $\mathrm{Ni}$ & Nickel & 0 & 0.17 & 0.2 \\
\hline 9 & $\mathrm{P}$ & Phosphorus & 0 & 0.05 & 0.06 \\
\hline 10 & S & Sulphur & 0 & 0.05 & 0.06 \\
\hline 11 & $\mathrm{Si}$ & Silicone & 0.3 & 0.70 & 0.8 \\
\hline
\end{tabular}

In this model, $\pi_{\text {tot }}^{a}$ is an intermediate variable. We denote by $\delta^{a}$ the set of feasible solutions to this problem.

Let us point out that the solution to this problem given by $\pi_{0}^{a}=1$ and $\pi^{a}=\left(\pi_{1}^{a}, \ldots, \pi_{N}^{a}\right)$ is not proportions. The proportions of premix and materials in the optimal corrected mixture are

$$
\tilde{\pi}_{i}^{a}=\frac{\pi_{i}^{a}}{z_{\text {weight }}^{\text {total }}\left(\pi^{a}\right)}=\frac{\pi_{i}^{a}}{1+z_{\text {weight }}\left(\pi^{a}\right)}
$$

for $i=0, \ldots, N$.

3.3. The Pareto Set. Problem $\left(P^{a}\right)$ is a problem with two criteria [10]: $z_{\text {cost }}\left(\pi^{a}\right)=c \pi^{a}$ and $z_{\text {weight }}\left(\pi^{a}\right)=u \pi^{a}$, where $c=\left(c_{1}, \ldots, c_{N}\right)$ and $u=(1, \ldots, 1)$. The feasible set in the criteria space $\mathbb{R}^{2}$ is defined by

$$
\begin{aligned}
\mathcal{S}_{c}^{a} & =\left\{z^{a}\left(\pi^{a}\right) \in \mathbb{R}^{2} \mid z^{a}\left(\pi^{a}\right)=C \pi^{a} \text { for } \pi^{a} \in \mathcal{S}^{a}\right\} \\
& =C \mathcal{S}^{a},
\end{aligned}
$$

where

$$
\begin{gathered}
z^{a}\left(\pi^{a}\right)=\left(\begin{array}{c}
z_{\text {cost }}\left(\pi^{a}\right) \\
z_{\text {weight }}\left(\pi^{a}\right)
\end{array}\right)=\left(\begin{array}{c}
\sum_{n=1}^{N} c_{n} \pi_{n}^{a} \\
\sum_{n=1}^{N} \pi_{n}^{a}
\end{array}\right)=C \pi^{a} \\
\text { with } C=\left(\begin{array}{ccc}
c_{1} & \cdots & c_{N} \\
1 & \cdots & 1
\end{array}\right) .
\end{gathered}
$$

A feasible solution $\pi^{a} \in \mathcal{S}^{a}$ is an efficient feasible solution if and only if there is no other feasible solution $\bar{\pi}^{a} \in \mathcal{S}^{a}$ such that

(i) $z_{i}\left(\bar{\pi}^{a}\right) \leq z_{i}\left(\pi^{a}\right)$ for every $i \in\{$ cost, weight $\}$;

(ii) $z_{j}\left(\bar{\pi}^{a}\right)<z_{j}\left(\pi^{a}\right)$ for at least one $j \in\{$ cost, weight $\}$, that is to say that no other feasible solution improves both criteria or at least one criterion without damaging the other criterion.
The set of efficient solution is the efficient set or the Pareto set denoted by $\mathscr{E}^{a}$. The efficient set or Pareto set in the criteria space is $\mathscr{C}_{c}^{a}=C_{\mathscr{C}}^{a}$. By considering the weighted sum of the two criteria using parameter $\lambda \in(0,1)$ and the problem

$$
\begin{aligned}
\min & z_{\lambda}\left(\pi^{a}\right) \\
& =(1-\lambda) z_{\text {cost }}\left(\pi^{a}\right)+\lambda z_{\text {weight }}\left(\pi^{a}\right) \\
\text { subject to } & \pi^{a} \in \mathcal{S}^{a},
\end{aligned}
$$

we can use the following relation for the Pareto set $\mathscr{E}^{a}[10]$ :

$$
\mathscr{E}^{a}=\bigcup_{\lambda \in(0,1)} \arg \min z_{\lambda}\left(\pi^{a}\right) .
$$

The efficient set $\mathscr{E}^{a}$ being a union of a finite number of faces of $\mathcal{S}^{a}$ follows that $\mathscr{C}_{c}^{a}$ is of the same form since it is the image of $\mathscr{E}^{a}$ by a linear transformation. As a subset of $\mathbb{R}^{2}, \mathscr{E}_{c}^{a}$ is formed by a finite number of pairwise segments connected by their endpoints, also called efficient vertices or efficient extreme points. It is a polygonal line. To each vertex of that polygonal line is associated an interval of values of $\lambda$, noted $[\underline{\lambda}, \bar{\lambda}]$. The union of all those intervals is $[0,1]$. Likewise, a unique value of $\lambda$ is assigned to each segment which corresponds to the common bound of the intervals of the two endpoints of the segment. See [11] for a complete description of $\mathscr{E}_{c}^{a}$.

3.4. Numerical Example. Table 5 shows the specifications of the premix. Note that these specifications satisfy the constraints of Type 1 but not the constraints of Type 2. Type 2 constraint coefficients are given in Table 6. The efficient vertices in the criteria space are given in Table 7 , and the corresponding optimal solutions in the decision space appear in Table 8. In these tables, the minimum cost solution is on the first line, while the minimum weight solution is on the last line. The other solutions correspond to intermediate vertices on the Pareto curve $\mathscr{E}_{c}^{a}$ given in Figure 3. This curve gives 
TABLE 6: Coefficients of Type 2 constraints.

\begin{tabular}{|c|c|c|c|c|c|c|c|c|c|c|}
\hline \multicolumn{11}{|c|}{$J=4$ constraint of Type 2} \\
\hline$j$ & 0 & 1 & 2 & 3 & 4 & 5 & 6 & 7 & 8 & $\beta_{j}$ \\
\hline $\begin{array}{l}1 \\
2\end{array}$ & 8.6944 & -1.3578 & 0 & -444.4444 & 8.2347 & 42.4085 & -36.160 & -0.4267 & 0 & $\begin{array}{l}\leq 9.2500 \\
\geq 7.6944\end{array}$ \\
\hline $\begin{array}{l}3 \\
4\end{array}$ & 55.0500 & 2.9106 & 0 & 800 & 120.3840 & 69.1392 & 65.088 & 0.7680 & 0 & $\begin{array}{l}\leq 54.0500 \\
\geq 51.2500\end{array}$ \\
\hline
\end{tabular}

TABLE 7: Efficient vertices in the criteria space $\mathbb{R}^{2}$.

\begin{tabular}{lccc}
\hline$\underline{\lambda}$ & $\bar{\lambda}$ & $z_{\text {cost }}^{a}$ & $z_{\text {weight }}^{a}$ \\
\hline 0 & 0.8402832 & 0.0028353 & 0.0195544 \\
0.8402832 & 0.9094854 & 0.0042987 & 0.0192762 \\
0.9094854 & 0.9791369 & 0.0049710 & 0.0192093 \\
0.9791369 & 1 & 0.0066641 & 0.0191732 \\
\hline
\end{tabular}

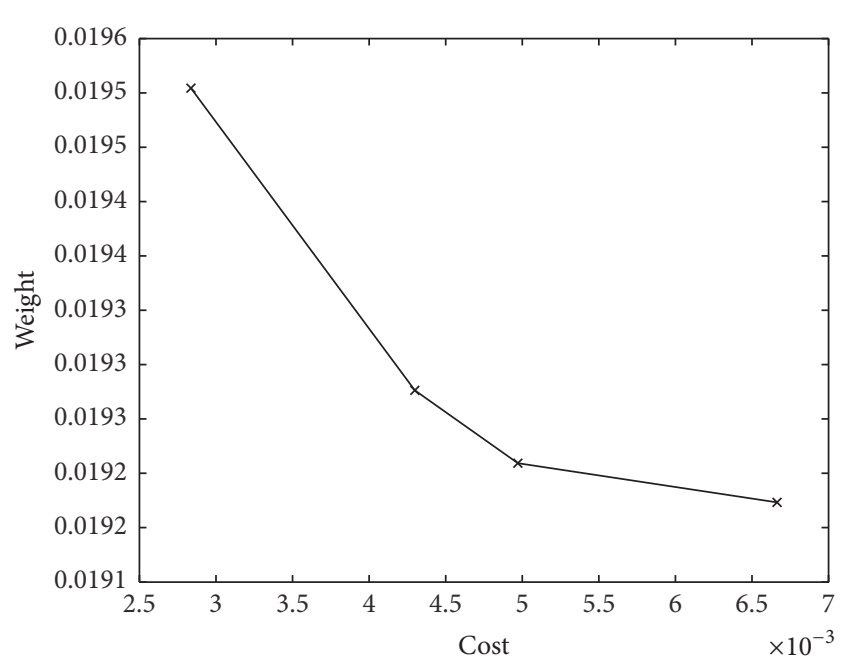

Figure 3: Pareto curve $\mathscr{E}_{c}^{a}$ in the criteria space $\mathbb{R}^{2}$.

us the best pairs $\left(z_{\text {cost }}, z_{\text {weight }}\right)$, going from the minimum cost efficient solution to the minimum weight efficient solution. For any point $\left(z_{\text {cost }}, z_{\text {weight }}\right) \in \mathscr{E}_{c}^{a}, z_{\text {cost }}$ is the minimum cost for all feasible correction $\pi^{a}$ such that $z_{\text {weight }}\left(\pi^{a}\right)=z_{\text {weight }}$, and, conversely, $z_{\text {weight }}$ is the minimum weight for all feasible correction $\pi^{a}$ such that $z_{\text {cost }}\left(\pi^{a}\right)=\mathrm{z}_{\text {cost }}$.

The solution of this problem shows that the correction at minimum cost effectively costs $0.0028353 \$$ and weights 0.0195544 weight units. The unit cost of the correction is then

$$
\frac{0.0028353 \$}{0.0195544 \text { weight unit }}=0.1449962 \frac{\$}{\text { weight unit }} \text {. }
$$

The corrected mixture formed thus weighs 1.0195543 weight units. On the other hand, the correction at minimum weight requires an addition of 0.0191732 weight unit, which costs
$0.0066641 \$$. In this case, the unit cost of the correction is

$$
\frac{0.0066641 \$}{0.0191732 \text { weight unit }}=0.3475736 \frac{\$}{\text { weight unit }} .
$$

The corrected mixture formed thus weighs 1.0191732 weight units.

The proportions of premix and materials in the optimal corrected mixture are reported in Table 9 for the efficient vertices.

Note that the correction obtained does not use materials $n=5$ and $n=6$; they could be removed. Recall that the effect of removing these two materials eliminates the possibility of finding a feasible mixture with only the available materials, without premix.

3.5. A Theoretical Result. We conclude this section by establishing a relation between the feasible sets $\delta$ and $\delta^{a}$. They are both closed subsets of $\mathbb{R}^{N}$, and they can be empty or nonempty. The set $\mathcal{S}$ can be nonempty while $\mathcal{S}^{a}$ is empty. When $\mathcal{S}$ is nonempty, it is bounded because

$$
\begin{aligned}
u^{t} \pi & =\sum_{n=1}^{N} \pi_{n}=1, \\
\pi_{n} \geq 0 &
\end{aligned}
$$

$$
\text { for }(n=1, \ldots, N) \text {. }
$$

When $\mathcal{S}^{a}$ is nonempty, it can be bounded (hence compact) or unbounded (thus not compact). The following result links these two situations. All possible situations are reported in Table 10.

Theorem 1. If $\mathcal{S}^{a}$ is nonempty, we have that

(i) $\mathcal{S}^{a}$ is bounded (thus compact) if and only if $\mathcal{S}$ is empty;

(ii) $\mathcal{S}^{a}$ is unbounded (thus not compact) if and only if $\mathcal{S}$ is nonempty.

Proof. If $\mathcal{S}^{a}$ is nonempty, let us take $0 \neq \pi \in \mathcal{S}$ and also take $\pi^{a} \in \mathcal{S}^{a}$. For $t \geq 0$, we set $\pi^{a}(t)=\pi^{a}+t \pi$. It is easily verified that $\pi^{a}(t) \in \mathcal{S}^{a}$ and thus $\mathcal{S}^{a}$ is unbounded. Conversely, if $\mathcal{S}^{a}$ is unbounded, there are $\pi^{a}(t) \in \mathcal{S}^{a}$ and $\pi \in \mathbb{R}^{N}$ such that $u^{t} \pi=1$ and $\pi^{a}(t)=\pi^{a}+t \pi \in \mathcal{S}^{a}$ (for all $t \geq 0$ ). Then we check that $\pi \in \mathcal{S}$ by substituting $\pi^{a}(t)$ in the constraint of $\left(P^{a}\right)$, dividing by $t$ and passing to the limit as $t$ goes to infinity. 
TABLE 8: Efficient vertices in the decision space $\mathbb{R}^{8}$.

\begin{tabular}{lcccccccc}
\hline$\pi_{0}^{a}$ & $\pi_{1}^{a}$ & $\pi_{2}^{a}$ & $\pi_{3}^{a}$ & $\pi_{4}^{a}$ & $\pi_{5}^{a}$ & $\pi_{6}^{a}$ & $\pi_{7}^{a}$ & 0 \\
1 & 0.0195543 & 0 & 0 & 0 & 0 & 0 & 0 & 0 \\
1 & 0.0126373 & 0 & 0 & 0 & 0 & 0 & 0.0066389 & 0.0066381 \\
1 & 0.0113950 & 0 & 0 & 0 & 0 & 0 & 0.0011760 \\
1 & 0.0124767 & 0 & 0 & 0 & 0 & 0 & 0.0066965 \\
\hline
\end{tabular}

TABLE 9: Proportions of premix and materials for efficient vertices.

\begin{tabular}{lcccccccc}
\hline$\tilde{\pi}_{0}^{a}$ & $\tilde{\pi}_{1}^{a}$ & $\tilde{\pi}_{2}^{a}$ & $\tilde{\pi}_{3}^{a}$ & $\tilde{\pi}_{4}^{a}$ & $\tilde{\pi}_{5}^{a}$ & $\tilde{\pi}_{6}^{a}$ & $\tilde{\pi}_{7}^{a}$ & 0 \\
\hline 0.9808207 & 0.0191793 & 0 & 0 & 0 & 0 & 0 & $\tilde{\pi}_{8}^{a}$ \\
0.9810883 & 0.0123983 & 0 & 0 & 0 & 0 & 0 & 0.0065133 \\
0.9811530 & 0.0111802 & 0 & 0 & 0 & 0 & 0 & 0.0065130 \\
0.9811875 & 0.0122420 & 0 & 0 & 0 & 0 & 0 & 0 & 0.0011538 \\
\hline
\end{tabular}

TABLE 10: Relationship between the feasible sets $\mathcal{S}$ and $\mathcal{S}^{a}$.

\begin{tabular}{ll}
\hline $\mathcal{S}$ & $\mathcal{S}^{a}$ \\
\hline Empty & $\begin{array}{l}\text { Empty } \\
\text { Nonempty, closed and } \\
\text { bounded (compact) }\end{array}$ \\
$\begin{array}{l}\text { Nonempty, closed and bounded } \\
\text { (compact) }\end{array}$ & empty \\
$\begin{array}{l}\text { Nonempty, closed and bounded } \\
\text { (compact) }\end{array}$ & $\begin{array}{l}\text { Nonempty, closed and } \\
\text { unbounded }\end{array}$ \\
\hline
\end{tabular}

\section{Correction to a Fixed Quantity of "Nonfree" Premix}

4.1. Problem Description. It would have been more realistic to consider that the premix has a possible nonzero cost in the preceding problem. So we reconsider the preceding problem assuming a possible nonzero unit $\operatorname{cost} c_{0}$ for the premix. We consider one criterion which is the unit cost of the corrected mixture.

Let us set

$$
\begin{aligned}
& \pi_{0}^{f} \geq 0 \quad \text { for the weight of the premix in the corrected mixture, } \\
& \pi_{n}^{f} \geq 0 \quad \text { for the weight of the } n \text {th material in the corrected mixture }(n=1, \ldots, \mathrm{N}) .
\end{aligned}
$$

As we want to correct a given weight of the premix, we set $\pi_{0}^{f}=1$, and the decision vector will be $\pi^{f}=\left(\pi_{1}^{f}, \ldots, \pi_{N}^{f}\right)$.

For the total weight, we have

$$
z_{\text {weight }}^{\text {total }}\left(\pi^{f}\right)=\sum_{n=0}^{N} \pi_{n}^{f}=1+\sum_{n=1}^{N} \pi_{n}^{f}=1+z_{\text {weight }}\left(\pi^{f}\right)
$$

because we have assumed that $\pi_{0}^{f}=1$, and the total cost is

$$
z_{\text {cost }}^{\text {total }}\left(\pi^{f}\right)=\sum_{n=0}^{N} c_{n} \pi_{n}^{f}=c_{0}+\sum_{n=1}^{N} c_{n} \pi_{n}^{f}=c_{0}+z_{\text {cost }}\left(\pi^{f}\right)
$$

which take into account the fact that the premix might have a nonzero cost, so $c_{0} \geq 0$.

For this problem, the criteria will be the unit cost of the corrected mixture, premix, and correction added together, given by the ratio

$$
\begin{aligned}
z^{f}\left(\pi^{f}\right) & =\frac{z_{\text {cost }}^{\text {total }}\left(\pi^{f}\right)}{z_{\text {weight }}^{\text {total }}\left(\pi^{f}\right)}=\frac{c_{0}+\sum_{n=1}^{N} c_{n} \pi_{n}^{f}}{1+\sum_{n=1}^{N} \pi_{n}^{f}} \\
& =\frac{c_{0}+z_{\text {cost }}\left(\pi^{f}\right)}{1+z_{\text {weight }}\left(\pi^{f}\right)} .
\end{aligned}
$$

4.2. Problem Formulation. The decision variables will be again $\pi_{n}^{f}$ for $n=1, \ldots, N$, and therefore the formulation is

$$
\begin{aligned}
& \min z^{f}\left(\pi^{f}\right)=\frac{c_{0}+\sum_{n=1}^{N} c_{n} \pi_{n}^{f}}{1+\sum_{n=1}^{N} \pi_{n}^{f}} \\
& \text { subject to } \pi^{f} \in \mathcal{S}^{f} .
\end{aligned}
$$

Let $\mathcal{S}^{f}$ be the set of feasible solution to this problem. Obviously, we have $\mathcal{S}^{f}=\mathcal{S}^{\mathrm{a}}$ because the constraints of this problem are the constraints of the preceding problem $\left(P^{a}\right)$.

4.3. Linear-Fractional Program. The problem $\left(P^{f}\right)$ is an example of a linear-fractional program. Under the assumption that the feasible region $\delta^{f}$ is nonempty and bounded, the Charnes-Cooper transformation [12]

$$
\begin{aligned}
& \eta_{0}=\frac{1}{1+\sum_{i=1}^{N} \pi_{i}^{f}}, \\
& \eta_{n}=\frac{\pi_{i}^{f}}{1+\sum_{i=1}^{N} \pi_{i}^{f}} \quad \text { for } n=1, \ldots, N,
\end{aligned}
$$


translates the linear-fractional program above to the equivalent linear program:

$$
\begin{aligned}
& \min \tilde{z}^{f}(\eta)=\sum_{n=0}^{N} c_{n} \eta_{n} \\
& \text { subject to (i) } \sum_{n=0}^{N} \eta_{n}=1 \text {; } \\
& \text { (ii) for } m=1, \ldots, M \text { : } \\
& \sum_{n=0}^{N} s_{m, n} \eta_{n} \leq \operatorname{spec}_{m}^{\max } \\
& \sum_{n=0}^{N} s_{m, n} \eta_{n} \geq \operatorname{spec}_{m}^{\min } \\
& \sum_{n=0}^{N}\left[\sum_{m=1}^{M} \alpha_{j, m} s_{m, n}\right] \eta_{n}\left\{\begin{array}{l}
\leq \\
= \\
\geq
\end{array}\right\} \beta_{j} \\
& \text { (iv) } \eta_{n} \geq 0 \text { for } n=0, \ldots, N \text {. }
\end{aligned}
$$

Let $\widetilde{\mathcal{S}}^{f}$ be the set of feasible solutions $\eta=\left(\eta_{0}, \ldots, \eta_{N}\right)$ of $\left(\widetilde{P}^{f}\right)$. Let us observe that $\left(\widetilde{P}^{f}\right)$ is similar to the first problem $(P)$, and the premix can now be considered as one of the available materials. The fact that we assume $\mathcal{S}^{a}$ nonempty and bounded, Theorem 1 implies that $(P)$ has no solution; hence, $\left(\widetilde{P}^{f}\right)$ has no solution $\eta=\left(\eta_{0}, \ldots, \eta_{n}\right) \in \widetilde{\mathcal{S}}^{f}$ with $\eta_{0}=0$. It follows that any solution of $\left(\widetilde{P}^{f}\right)$, if it exists, is such that $\eta_{0}>0$ and that the corresponding solution of $\left(P^{f}\right)$ is given by

$$
\pi_{n}^{f}=\frac{\eta_{n}}{\eta_{0}} \quad \text { for } n=0, \ldots, N
$$

and hence $\pi_{0}^{f}=1$. In case the feasible region $\mathcal{S}^{a}$ is nonempty and unbounded, $\left(\widetilde{P}^{f}\right)$ might have a solution $\eta=\left(\eta_{0}, \ldots, \eta_{n}\right) \in$ $\widetilde{\mathcal{S}}^{f}$ with $\eta_{0}>0$; then the solution of $\left(P^{f}\right)$ is again given by the preceding formula. But it might happen that $\left(\widetilde{P}^{f}\right)$ has a solution $\eta=\left(\eta_{0}, \ldots, \eta_{n}\right) \in \widetilde{\mathcal{S}}^{f}$ with $\eta_{0}=0$; then the resulting mixture is not a corrected mixture. It means that it is better to use original materials $(n=1, \ldots, N)$ to produce the desired mixture because it is cheaper than trying to use the premix. Hence,

$$
\pi_{n}=\eta_{n} \quad \text { for } n=1, \ldots, N,
$$

is a solution of the original problem $(P)$. In this case, let us define $\pi^{f}$ by the formula $\pi_{0}^{f}=0$, and $\pi_{n}^{f}=\eta_{n}$ for $n=$ $0, \ldots, N$, as before.

4.4. A Parametric Analysis. We could be interested by the variation of the criteria, the unit cost of the resulting (corrected or not) mixture, with respect to the cost $c_{0}$. This information is obtained from a parametric analysis of the criteria with respect to the parameter $c_{0}$. We split the criteria of $\left(\widetilde{P}^{f}\right)$ in two parts:

$$
\widetilde{z}^{f}(\eta)=\sum_{n=1}^{N} c_{n} \eta_{n}+c_{0} \eta_{0}=\widetilde{z}_{1}^{f}(\eta)+c_{0} \widetilde{z}_{2}^{f}(\eta)
$$

and we consider the following bicriteria problem:

$$
\begin{array}{cl}
\min & \widetilde{z}_{1}^{f}(\eta)=\sum_{n=1}^{N} c_{n} \eta_{n} \\
\min & \widetilde{z}_{2}^{f}(\eta)=\eta_{0} \\
\text { subject to } & \eta \in \widetilde{\mathcal{S}}^{f} .
\end{array}
$$

The Pareto curve of this problem, obtained by considering the weighted sum, is

$$
\widetilde{z}_{\lambda}^{f}(\eta)=(1-\lambda) \widetilde{z}_{1}^{f}(\eta)+\lambda \widetilde{z}_{2}^{f}(\eta)
$$

and the correspondence

$$
\begin{aligned}
\lambda & =\frac{c_{0}}{1+c_{0}} \\
\text { or } c_{0} & =\frac{\lambda}{1-\lambda},
\end{aligned}
$$

allows us to find a piecewise linear continuous increasing concave representation for the optimal minimal unit cost $z_{*}^{f}\left(c_{0}\right)=\min z^{f}\left(\pi^{f}\right)$ in terms of $c_{0}$.

Since

$$
\begin{aligned}
& z_{\text {cost }}\left(\pi^{f}\right)=\sum_{n=1}^{N} c_{n} \pi_{n}^{f} \\
& = \begin{cases}\frac{1}{\eta_{0}} \sum_{n=1}^{N} c_{n} \eta_{n}=\frac{1}{\eta_{0}} \widetilde{z}_{1}^{f}(\eta) & \text { if } \eta_{0}>0\left(\text { or } \pi_{0}^{f}=1\right), \\
\sum_{n=1}^{N} c_{n} \eta_{n}=\widetilde{z}_{1}^{f}(\eta) & \text { if } \eta_{0}=0\left(\text { or } \pi_{0}^{f}=0\right),\end{cases} \\
& z_{\text {weight }}\left(\pi^{f}\right)=\sum_{n=1}^{N} \pi_{n}^{f} \\
& = \begin{cases}\frac{1}{\eta_{0}} \sum_{n=1}^{N} \eta_{n}=\frac{1}{\eta_{0}}\left(1-\eta_{0}\right) & \text { if } \eta_{0}>0\left(\text { or } \pi_{0}^{f}=1\right), \\
\sum_{n=1}^{N} \eta_{n}=1 & \text { if } \eta_{0}=0\left(\text { or } \pi_{0}^{f}=0\right),\end{cases}
\end{aligned}
$$

we have

$$
z_{*}^{f}\left(c_{0}\right)=\frac{c_{0} \pi_{0}^{f}+z_{\text {cost }}\left(\pi^{f}\right)}{\pi_{0}^{f}+z_{\text {weight }}\left(\pi^{f}\right)}
$$

that is, valid not only for $\pi_{0}^{f}=1$ but also for $\pi_{0}^{f}=0$ and where $\pi^{f}$ is the optimal solution. 
TABLE 11: Efficient vertices of $\left(\widetilde{P}^{f s}\right)$ and $\left(P^{f}\right)$ in the criteria space $\mathbb{R}^{2}$.

(a)

\begin{tabular}{lccc}
\hline$\underline{\lambda}$ & $\bar{\lambda}$ & $\widetilde{z}_{1}^{f}(\eta)$ & $\widetilde{z}_{2}^{f}(\eta)$ \\
\hline 0 & 0.124887 & 0.002781 & 0.980820 \\
0.124887 & 0.127242 & 0.005732 & 0.960140 \\
0.127242 & 0.249832 & 0.011545 & 0.920268 \\
0.249832 & 0.343079 & 0.024633 & 0.880968 \\
0.343079 & 0.604078 & 0.045238 & 0.841514 \\
0.604078 & 0.710795 & 0.045834 & 0.841124 \\
0.710795 & 0.874033 & 0.056158 & 0.836911 \\
0.874033 & 1 & 0.056666 & 0.836838 \\
\hline
\end{tabular}

(b)

\begin{tabular}{lccc}
\hline$\underline{c}_{0}$ & $\bar{c}_{0}$ & $z_{\text {cost }}^{f}\left(\pi^{f}\right)$ & $z_{\text {weignt }}^{f}\left(\pi^{f}\right)$ \\
\hline 0 & 0.142710 & 0.002835 & 0.019554 \\
0.142710 & 0.145794 & 0.005970 & 0.041513 \\
0.145794 & 0.333035 & 0.012545 & 0.086638 \\
0.333035 & 0.522255 & 0.027961 & 0.135113 \\
0.522255 & 1.525752 & 0.053758 & 0.188333 \\
1.525752 & 2.450600 & 0.054491 & 0.188885 \\
2.450600 & 6.938604 & 0.067101 & 0.194869 \\
6.938604 & $+\infty$ & 0.067714 & 0.194974 \\
\hline
\end{tabular}

TABLE 12: Optimal solutions of $\left(\widetilde{P}^{f s}\right)$ and $\left(P^{f}\right)$ in the decision space $\mathbb{R}^{7}$.

(a)

\begin{tabular}{|c|c|c|c|c|c|c|c|c|}
\hline$\eta_{0}$ & $\eta_{1}$ & $\eta_{2}$ & $\eta_{3}$ & $\eta_{4}$ & $\eta_{5}$ & $\eta_{6}$ & $\eta_{7}$ & $\eta_{8}$ \\
\hline 0.980820 & 0.019179 & 0 & 0 & 0 & - & - & 0 & 0 \\
\hline 0.960140 & 0.038506 & 0 & 0.001352 & 0 & - & - & 0 & 0 \\
\hline 0.920268 & 0.079282 & 0 & 0.000448 & 0 & - & - & 0 & 0 \\
\hline 0.880968 & 0.098547 & 0 & 0 & 0.020483 & - & - & 0 & 0 \\
\hline 0.841514 & 0.096656 & 0 & 0 & 0.061829 & - & - & 0 & 0 \\
\hline 0.841124 & 0.094791 & 0 & 0 & 0.062039 & - & - & 0.002044 & 0 \\
\hline 0.836911 & 0.081505 & 0 & 0 & 0.064288 & - & - & 0.001878 & 0.015415 \\
\hline 0.836838 & 0.081852 & 0 & 0 & 0.064325 & - & - & 0 & 0.016983 \\
\hline
\end{tabular}

(b)

\begin{tabular}{|c|c|c|c|c|c|c|c|c|}
\hline$\pi_{0}^{f}$ & $\pi_{1}^{f}$ & $\pi_{2}^{f}$ & $\pi_{3}^{f}$ & $\pi_{4}^{f}$ & $\pi_{5}^{f}$ & $\pi_{6}^{f}$ & $\pi_{7}^{f}$ & $\pi_{8}^{f}$ \\
\hline 1 & 0.019554 & 0 & 0 & 0 & - & - & 0 & 0 \\
\hline 1 & 0.040105 & 0 & 0.001408 & 0 & - & - & 0 & 0 \\
\hline 1 & 0.086151 & 0 & 0.000486 & 0 & - & - & 0 & 0 \\
\hline 1 & 0.111862 & 0 & 0 & 0.023251 & - & - & 0 & 0 \\
\hline 1 & 0.114859 & 0 & 0 & 0.073473 & - & - & 0 & 0 \\
\hline 1 & 0.112696 & 0 & 0 & 0.073758 & - & - & 0.002430 & 0 \\
\hline 1 & 0.097389 & 0 & 0 & 0.076816 & - & - & 0.002244 & 0.018420 \\
\hline 1 & 0.097811 & 0 & 0 & 0.076867 & - & - & 0 & 0.020295 \\
\hline
\end{tabular}

4.5. Numerical Example. We consider two situations with the preceding data.

Case 1. We use all the materials, without $n=5$ and $n=6$. In this case, $\mathcal{\delta}$ is empty and $\mathcal{S}^{a}$ is nonempty and bounded; hence $\eta_{0}$ will always be strictly positive. Tables 11 and 12 present results for $\left(\widetilde{P}^{f s}\right)$ and the corresponding values for $\left(P^{f}\right)$.

Case 2. We consider all the $N=8$ materials; then $\mathcal{S}$ is nonempty and $\mathcal{S}^{a}$ is nonempty and not bounded; hence it 
TABLE 13: Efficient vertices of $\left(\widetilde{P}^{f s}\right)$ and $\left(P^{f}\right)$ in the criteria space $\mathbb{R}^{2}$.

(a)

\begin{tabular}{lccr}
\hline$\underline{\lambda}$ & $\bar{\lambda}$ & $\widetilde{z}_{1}^{f}(\eta)$ & $\widetilde{z}_{2}^{f}(\eta)$ \\
\hline 0 & 0.124887 & 0.002781 & 0.980820 \\
0.124887 & 0.127242 & 0.005732 & 0.960140 \\
0.127242 & 0.249832 & 0.011545 & 0.920267 \\
0.249832 & 0.261550 & 0.024633 & 0.880970 \\
0.261550 & 0.270235 & 0.153784 & 0.516323 \\
0.270235 & 1 & 0.344983 & 0 \\
\hline
\end{tabular}

(b)

\begin{tabular}{lccc}
\hline$\underline{c}_{0}$ & $\bar{c}_{0}$ & $z_{\text {cost }}^{f}\left(\pi^{f}\right)$ & $z_{\text {weignt }}^{f}\left(\pi^{f}\right)$ \\
\hline 0 & 0.142710 & 0.002835 & 0.019554 \\
0.142710 & 0.145794 & 0.005970 & 0.041513 \\
0.145794 & 0.333035 & 0.012545 & 0.086640 \\
0.333035 & 0.354188 & 0.027961 & 0.135112 \\
0.354188 & 0.370304 & 0.297848 & 0.936768 \\
0.370304 & $+\infty$ & 0.344984 & 1 \\
\hline
\end{tabular}

TABLE 14: Optimal solutions of $\left(\widetilde{P}^{f s}\right)$ and $\left(P^{f}\right)$ in the decision space $\mathbb{R}^{9}$.

(a)

\begin{tabular}{lccccccc}
\hline$\eta_{0}$ & $\eta_{1}$ & $\eta_{2}$ & $\eta_{3}$ & $\eta_{4}$ & $\eta_{5}$ & $\eta_{6}$ & $\eta_{7}$ \\
\hline 0.980820 & 0.019179 & 0 & 0 & 0 & 0 & 0 & 0 \\
0.960140 & 0.038506 & 0 & 0.001352 & 0 & 0 & 0 & 0 \\
0.920267 & 0.079284 & 0 & 0.000448 & 0 & 0 & 0 & 0 \\
0.880970 & 0.098547 & 0 & 0 & 0.020482 & 0 & 0 & 0 \\
0.516323 & 0.277318 & 0 & 0 & 0.004485 & 0.272517 & 0.192517 & 0 \\
0 & 0.530479 & 0 & & 0 & 0 & 0 & 0 \\
\hline
\end{tabular}

(b)

\begin{tabular}{cccccccc}
\hline$\pi_{0}^{f}$ & $\pi_{1}^{f}$ & $\pi_{2}^{f}$ & $\pi_{3}^{f}$ & $\pi_{4}^{f}$ & $\pi_{5}^{f}$ & $\pi_{6}^{f}$ & $\pi_{7}^{f f}$ \\
\hline 1 & 0.019554 & 0 & 0 & 0 & 0 & $\pi_{8}^{f}$ \\
1 & 0.040104 & 0 & 0.001408 & 0 & 0 & 0 & 0 \\
1 & 0.086153 & 0 & 0.000486 & 0 & 0 & 0 & 0 \\
1 & 0.111862 & 0 & 0 & 0.023249 & 0 & 0 & 0 \\
1 & 0.537102 & 0 & 0 & 0.292976 & 0.106690 & 0 & 0 \\
0 & 0.530479 & 0 & 0.004485 & 0.272517 & 0.192517 & 0 & 0 \\
\hline
\end{tabular}

might happen that $\eta_{0}$ be 0 for large values of $c_{0}$. Tables 13 and 14 present results for $\left(\widetilde{P}^{f_{s}}\right)$ and the corresponding values for $\left(P^{f}\right)$.

Let us observe that the results for $c_{0}=0$ correspond to the results obtained in Section 3.

\section{Conclusion}

Three simple mixture problems occurring in metallurgy were analyzed. We first considered the basic mixture problem. We then considered a correction problem to a "free" premix. We have shown that, in the case of a free premix, the minimal cost correction does not generally correspond to the minimum weight correction. We built the Pareto curve that gives all intermediate values between these two extremes. The same problem, with a "nonfree" premix, leads to a unique minimal unit cost solution.

\section{Competing Interests}

The authors declare that there is no conflict of interests regarding the publication of this paper.

\section{Acknowledgments}

This work has been financially supported by an individual discovery grant from NSERC (National Sciences and Engineering Research Council of Canada) for the first author. 


\section{References}

[1] G. B. Dantzig, Linear Programming and Extensions, Princeton University Press, Princeton, NJ, USA, 1963.

[2] G. B. Dantzig, “The diet problem," Interfaces, vol. 20, no. 4, pp. 43-47, 1990.

[3] F. Dubeau, P.-O. Julien, and C. Pomar, "Étude ce cas: un porcin dans un environnement sain," INFOR, vol. 46, pp. 199-217, 2008.

[4] S. G. Garille and S. I. Gass, "Stigler's diet problem revisited," Operations Research, vol. 49, no. 1, pp. 1-13, 2001.

[5] L. M. Lancaster, "The evolution of the diet model in managing food systems," Interfaces, vol. 22, no. 5, pp. 59-68, 1992.

[6] L. M. Lancaster, "The history of the application of mathematical programming to menu planning," European Journal of Operational Research, vol. 57, no. 3, pp. 339-347, 1992.

[7] G. J. Stigler, “The cost of subsistence," American Journal of Agricultural Economics, vol. 27, no. 2, pp. 303-314, 1945.

[8] C. Audet, J. Brimberg, P. Hansen, S. Le Digabel, and N. Mladenović, "Pooling problem: alternate formulations and solution methods," Management Science, vol. 50, no. 6, pp. 761-776, 2004.

[9] A. Rong and R. Lahdelma, "Fuzzy chance constrained linear programming model for optimizing the scrap charge in steel production," European Journal of Operational Research, vol. 186, no. 3, pp. 953-964, 2008.

[10] R. E. Steuer, Multiple Criteria Optimization, Wiley Series in Probability and Mathematical Statistics: Applied Probability and Statistics, John Wiley \& Sons, New York, NY, USA, 1986.

[11] F. Dubeau and A. Kadri, "Computation and visualization of the Pareto set in the criterion space for the bicriteria linear programming problem," International Journal of Mathematics and Computation, vol. 15, no. 2, pp. 1-15, 2012.

[12] A. Charnes and W. W. Cooper, "Programming with linear fractional functionals," Naval Research Logistics Quarterly, vol. 9, pp. 181-186, 1962. 


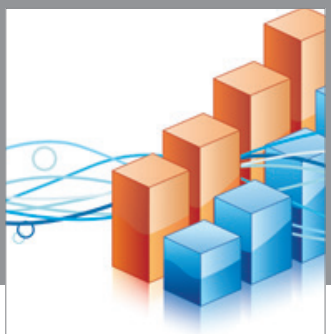

Advances in

Operations Research

vatem alat4

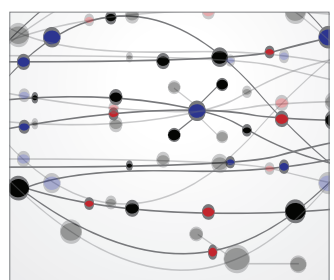

\section{The Scientific} World Journal
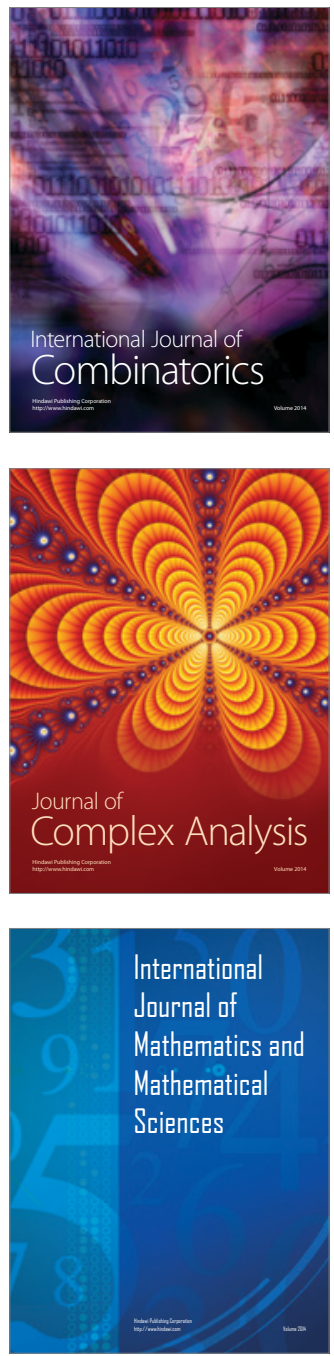
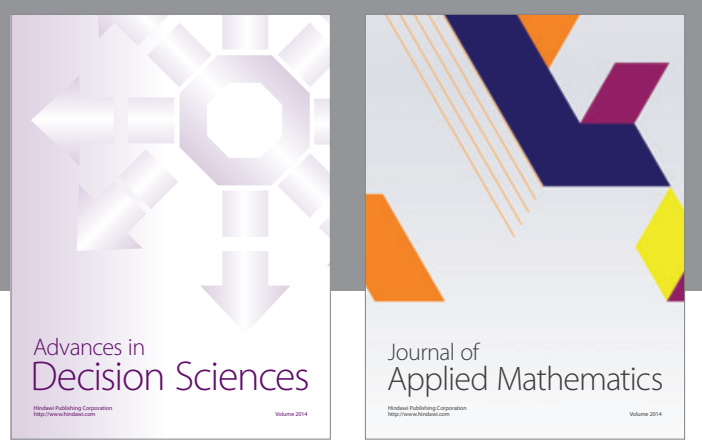

Algebra

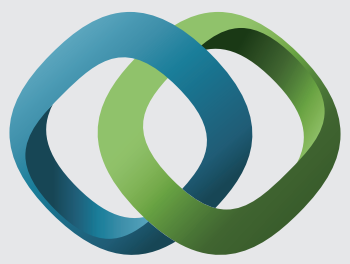

\section{Hindawi}

Submit your manuscripts at

https://www.hindawi.com
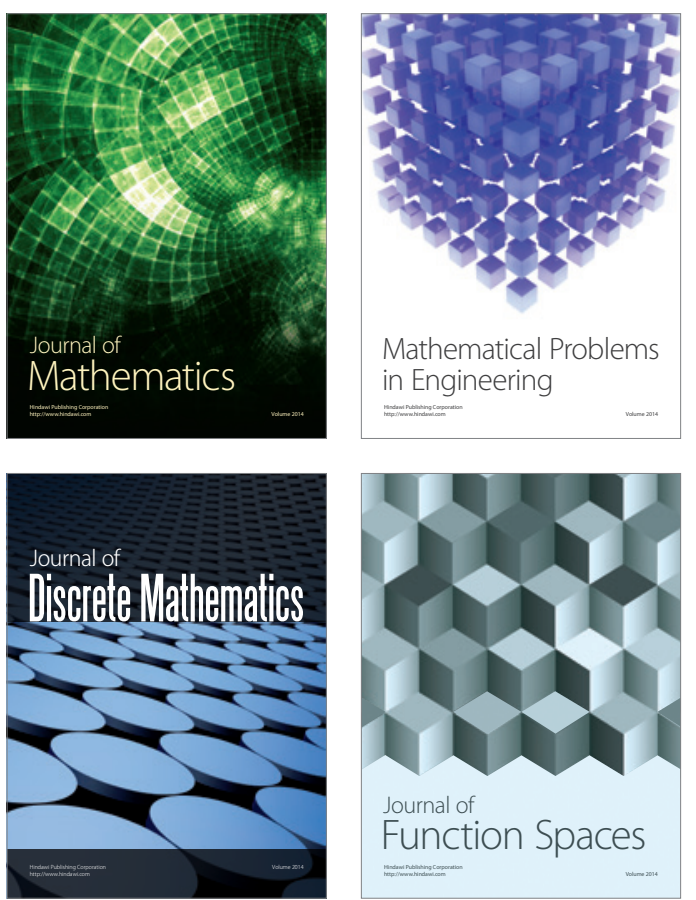

Mathematical Problems in Engineering
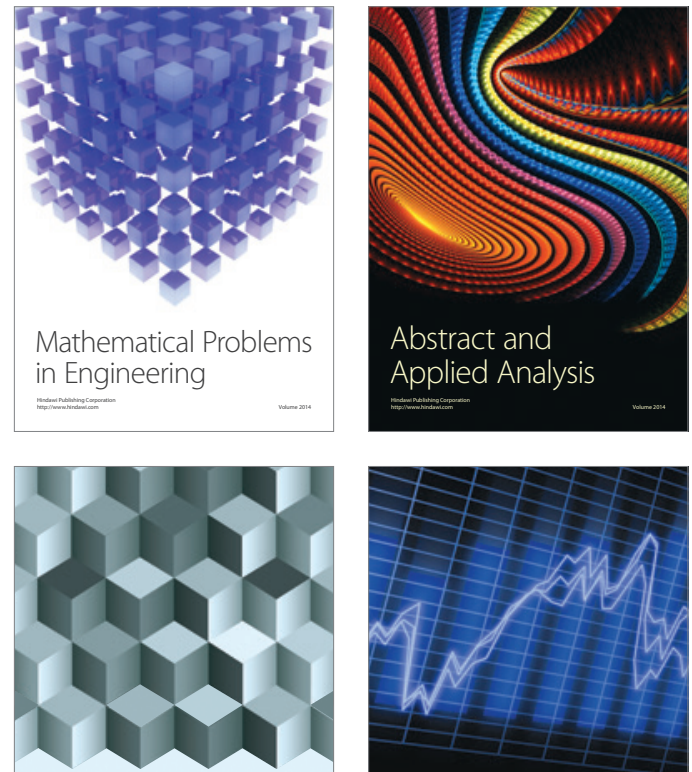

Journal of

Function Spaces

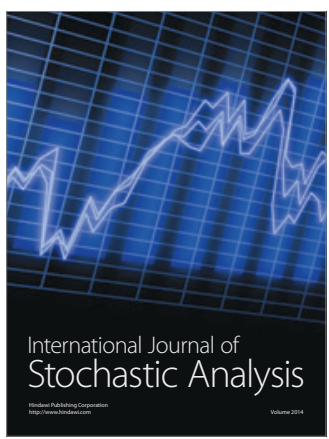

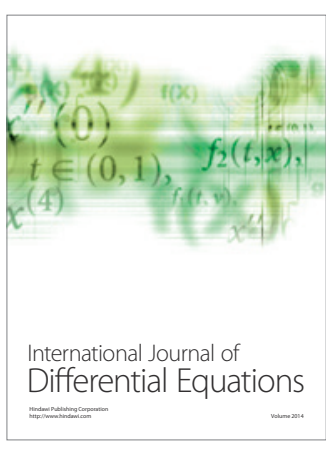
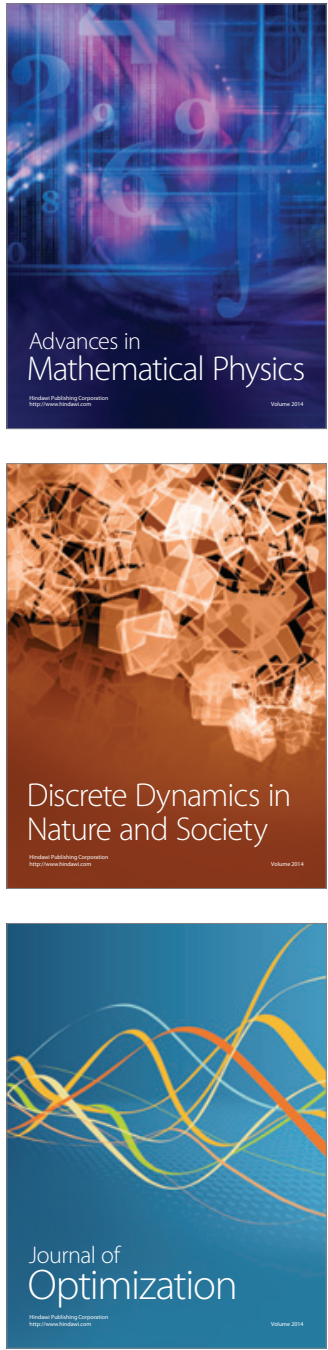